\title{
Business Process Modeling Languages: A Comparative Framework
}

\author{
José Luís Pereira ${ }^{1}$ and Diogo Silva ${ }^{1}$, \\ ${ }^{1}$ Universidade do Minho, Departamento de Sistemas de Informação, \\ Campus de Azurém, Guimarães, Portugal \\ jlmp@dsi.uminho.pt, a58899@alunos.uminho.pt
}

\begin{abstract}
Underlying any Business Process Management (BPM) project is the need to represent business processes, using an appropriate language. In this paper, based on a thorough review of the relevant literature, we made a comparative analysis of five business process modeling languages, widely used in the context of BPM projects. The main objective is to understand the strengths and major limitations of each one, in order to draw a comparative perspective between them. For this purpose, we have created a comparative framework in which each one of the languages are characterized regarding a number of relevant criteria. Finally, a prototype specifically developed to support this framework, is presented. The purpose of this prototype is to assist users in choosing a suitable business process modeling language, according to their specific needs.
\end{abstract}

Keywords: Business Process Management, Business Process Modeling, Process Modeling Languages.

\section{Introduction}

This paper aims to analyze and compare some of the major languages for modeling business processes, which are used in the context of Business Process Management (BPM) projects in organizations. The set of business process modeling languages in use today is already quite extensive, which creates difficulties to modelers when they need to choose one of them to use in their BPM projects. These projects are increasingly valued by organizations, as they need to improve their business processes, thus ensuring the adequate implementation of their business strategies, a better alignment between those strategies and their IS/IT solutions and, in general, improving their business management capabilities [1].

Among the various existing languages for business process modeling, there are five which are the most influential these days: the BPMN language (Business Process Model and Notation), currently in version 2.0, is the most widely used today and considered by some as the standard [2]; the EPC (Event-driven Process Chain), used within the well-known ARIS toolset [3]; the UML-AD (Unified Modelling Language - Activity Diagrams), created by the OMG (Object Management Group), initially to support the development of software, and recently with a more widespread context of 
use [4]; the IDEF (Integration DEFinition), also known for being a family of languages with several distinct purposes [5]; and finally the RAD (Role Activity Diagram) language, with a special focus on the participants in a business process, manly their interactions [6].

Among the panoply of languages for business process modeling that exist today it is important to clarify the aspects in which they are distinct, highlighting their strengths and limitations. Thus, allowing for a more informed choice by business analysts (who use them to document and define processes), or by users (who have to validate the models built by the former), when it is necessary to choose a language.

Concerning the structure of this paper, first we identify and describe the set of criteria for characterizing modeling languages that our literature review, and our own experience in the field, has pointed out as the most relevant. Next, we evaluate each one of the five business process modeling languages against the mentioned set of criteria, creating a comparative framework. Finally, we present a prototype of a system that implements the comparative framework. This prototype has been prepared to receive and incorporate feedback from experienced business process modelers and analysts in order to improve the contents of the comparative framework. The objective is to assist users in choosing the most suitable process modeling language, taking into account the specificities of the BPM project they have in hands.

\section{Characterization of Business Process Modeling Languages}

The modeling of business processes has a great impact on the success of any BPM project. Therefore, the choice of the business process modeling language to use in a particular BPM project is very important and should depend on its specific objectives. For instance, the purpose of the project is:

- Modeling of business processes for simple documentation, regarding its communication and dissemination among stakeholders?

- Modeling of business processes to support the optimization of its operation?

- Modeling of business processes regarding their implementation using IT?

On the other hand, the choice of the business process modeling language to use should also take into account the characteristics of the processes themselves, such as:

- Involving predominantly human resources?

- Or integrating automated processing systems?

- Consisting of well-structured workflows?

- Or involving predominantly interactions among people?

Nowadays, the offer in terms of languages/notations for business process modeling is considerable and, although all of them share the same goal - to model business processes - each one has its own specificities. The five languages that we have selected for this study, although being representative of what one can find in this area, have different characteristics, strengths and limitations. Thus, it is important to have a mechanism to compare them in order to systematize their differences and similarities. To this end we have carried out a thorough review of the relevant literature which focuses on the characterization of process modeling languages. 
Different authors use distinct criteria to evaluate business process modeling languages, although some criteria tend to receive more attention in the literature:

- Expressiveness - the capability of the language to represent the many different organizational situations, both in terms of the behavioral, functional, structural or informational perspectives;

- Readability - the greater or lesser ease for people to understand and interpret the process models defined with the language;

- Usability - although related with readability, this criterion focuses mainly on the complexity of the use of the language;

- User Friendly - also somewhat related to readability, this criterion refers to a greater or lesser attractiveness of the language, in the sense that its use can be more or less pleasant and intuitive;

- Formality - the rigor with which the semantics of the language is defined, reducing/removing the ambiguities in the interpretation of the models;

- Versatility - if the language is suitable only for the documentation and analysis of processes, or does it allows the execution/simulation of models;

- Universality - the greater or lesser disclosure of the language among users, with implications in terms of support for their use;

- Tools Support - the greater or lesser availability of suitable tools to support the use of the language;

- Flexibility - the greater or lesser capability of the elements of a language to be used in different scenarios and to represent distinct features.

The following table (Table 1) systematizes the criteria mentioned above as well as the authors found in the literature that used those criteria to compare business process modeling languages.

Table 1. Comparison Criteria vs Process Languages: Coverage in the existing literature.

\begin{tabular}{|l|l|l|l|l|l|}
\hline $\begin{array}{l}\text { Languages } / \\
\text { Criteria }\end{array}$ & \multicolumn{1}{|c|}{ BPMN } & \multicolumn{1}{|c|}{ EPC } & \multicolumn{1}{|c|}{ UML-AD } & \multicolumn{1}{|c|}{ RAD } & \multicolumn{1}{|c|}{ IDEF } \\
\hline Expressiveness & $\begin{array}{l}{[7],[8],[9],} \\
{[10],[11],[12]}\end{array}\left[\begin{array}{l}{[10],[11]} \\
{[1],[9],}\end{array}\right.$ & $\begin{array}{l}{[7],[8],[9],} \\
{[10],[11],}\end{array}$ & $\begin{array}{l}{[7],[8],[10],} \\
{[12]}\end{array}$ & $\begin{array}{l}{[7],[8],[9],} \\
{[10]}\end{array}$ \\
\hline Readability & {$[11],[12],[13]$} & {$[11],[13]$} & {$[11],[13]$} & {$[12],[13]$} & {$[12],[13]$} \\
\hline Usability & {$[12],[13]$} & {$[13]$} & {$[13]$} & {$[12],[13]$} & {$[13]$} \\
\hline User Friendly & {$[12]$} & & & {$[12]$} & \\
\hline Formality & {$[10]$} & {$[10]$} & {$[10]$} & {$[10]$} & {$[10]$} \\
\hline Versatility & {$[10]$} & {$[10]$} & {$[10]$} & {$[10]$} & {$[10]$} \\
\hline Universality & {$[11]$} & {$[11]$} & {$[11]$} & & \\
\hline Tools Support & {$[11]$} & {$[11]$} & {$[11]$} & & \\
\hline Flexibility & {$[12]$} & & & {$[12]$} & \\
\hline
\end{tabular}


Unfortunately, as Table 1 illustrates, there are few examples of authors who have compared all the five languages against the same criterion. The exceptions are [7], [8] and [10] (regarding the expressiveness criterion), [13] (regarding the usability criterion) and [10], (regarding the formality and versatility criteria).

As one can see, the expressiveness of the process modeling language is, by far, the one that has attracted more attention until now. The other eight criteria have had some attention, but not as much as the expressiveness one.

To these nine criteria we have added a few more, as a result of our own experience in the business process modeling field:

- Concision - the greater or lesser capability of the language to represent the various facets of a business process using a smaller set of elements;

- Ease of Learning - the greater or lesser effort required to master and be productive in the use of language;

- Innovation Inducer - the greater or lesser ease with which the language induces modelers to discover new solutions and modeling practices;

- Evolutionary - directly linked to the probability of a language to be updated and improved in the future;

- Collaborative Work - the greater or lesser suitability of the language to support the modeling of collaborative work situations in a process (e.g. meetings).

Regarding these five criteria, we simply were not able to find any reference to them in the research literature dedicated to the comparison of process modeling languages. These clearly represent "work to do" that deserve and will have our attention in the near future.

\section{Evaluation of the Business Process Modeling Languages}

Once identified the criteria for the comparative analysis of business process modeling languages we have evaluated the selected five languages against those criteria, taking into consideration the opinions produced by the authors that have made comparisons (Table 1), filtered by our own opinions as users of those process modeling languages.

In the following table (Table 2) we systematize the results of our study. Each cell of the table represents a question like "How do you evaluate the language $\boldsymbol{X}$ regarding its support of the criterion $\boldsymbol{Y}$ ?" receiving a value in a scale from 0 (meaning that language $\mathbf{X}$ do not support the criterion $\mathbf{Y}$ ) to 5 (meaning that language $\mathbf{X}$ fully support the criterion $\mathbf{Y}$ ).

As Table 2 illustrates, while there are criteria which every business process modeling language supports, although with different capabilities, there are also some criteria which are very distinctly supported by different modeling languages, with values from 0 (no support) to 5 (full support). Regarding modeling languages, the IDEF family is the one with major limitations, while BPMN stands out as the language that offers the best support in the majority of the criteria, which is not surprising considering its widespread use in the area of business processes modeling. 
Table 2. Process Modeling Languages vs Criteria: Evaluation.

\begin{tabular}{|c|c|c|c|c|c|}
\hline $\begin{array}{l}\text { Languages / } \\
\text { Criteria }\end{array}$ & BPMN & EPC & UML-AD & RAD & IDEF \\
\hline Expressiveness & 4 & 3 & 4 & 3 & 2 \\
\hline Readability & 5 & 4 & 4 & 4 & 3 \\
\hline Usability & 4 & 4 & 4 & 4 & 3 \\
\hline User Friendly & 5 & 5 & 5 & 5 & 3 \\
\hline Formality & 5 & 5 & 5 & 1 & 5 \\
\hline Versatility & 5 & 5 & 4 & 3 & 3 \\
\hline Universality & 5 & 4 & 5 & 3 & 3 \\
\hline Tools Support & 5 & 2 & 5 & 2 & 3 \\
\hline Flexibility & 4 & 4 & 4 & 4 & 3 \\
\hline Concision & 4 & 4 & 4 & 4 & 3 \\
\hline Ease of Learning & 5 & 5 & 5 & 4 & 3 \\
\hline $\begin{array}{l}\text { Innovation } \\
\text { Inducer }\end{array}$ & 4 & 4 & 3 & 5 & 2 \\
\hline Evolutionary & 4 & 4 & 4 & 2 & 3 \\
\hline $\begin{array}{l}\text { Collaborative } \\
\text { Work }\end{array}$ & 2 & 2 & 2 & 5 & 0 \\
\hline
\end{tabular}

Expressiveness was the criterion that best allowed a consolidated comparison between the five process modeling languages, due to its coverage by several authors. In general, they argue that the IDEF family of languages (in particular IDEF0 and IDEF3) is unable to model the organizational context in which business processes exist and therefore are quite limited in their expressiveness. Regarding the BPMN and UML-AD languages, both exhibit more expressiveness as they allow us to represent the four essential perspectives of business processes (organizational, functional, behavioral and informational), standing out from the other two process modeling languages - EPC and RAD.

Readability is another criterion that has also been treated by some authors. In general all of them argue that BPMN is the language that best serves the two communities interested in business process modeling - business analysts and IT specialists - as BPMN models can easily be understood by both, which is a plus. This fact distinguish and justify the maximum valuation of BPMN in this criterion. On the contrary, the low level elements of IDEF leaves this language again in last place. 
Regarding usability, due to the scarcity of literature comparing process modeling languages against this criterion, its assessment was carried out manly based on the author's experience. This is a criterion in which there is a balance between the different process modeling languages, with IDEF showing again some difficulties, in particular when we have to deal with more complex processes.

While IDEF is also the least interesting modeling language regarding the user friendly criterion, based in our experience with the other four modeling languages we claim that they are equally friendly, and very simple and pleasant to use. So, they earn the maximum value in this criterion.

Concerning the formality criterion, noticeably there is a modeling language in clear inferiority when compared to the other languages - RAD. This is a language that, contrarily to all the others, does not have a formal definition of its semantics, nor a standardized representation of its elements.

In the versatility criterion once again the BPMN and EPC languages stand out. These two modeling languages, besides allowing the documentation and analysis of business processes, at the present, are the only ones that may have their models directly executed by BPMS (Business Process Management System), without requiring any translation or mapping to another language. UML-AD is making the same route but, for now, is not already there. At the present, RAD and IDEF's only allow the development of models for documentation and analysis purposes.

In the universality criterion, maybe due to their affiliation to the OMG, the BPMN and UML-AD languages are the most visible and recognized at a global level. In this context EPC, although the fact that it is limited to the ARIS family of tools, also has a significant level of recognition at a worldwide level. In the case of RAD and IDEF, their user base is very limited.

Regarding tools support, also as a reason for or as a consequence of the previous criterion, BPMN and UML-AD languages have the large base of tools to support those languages. At a lower level of tools support stands IDEF and at an even lower level appears RAD. The case of EPC is very singular because, being a proprietary language of the ARIS family, the tools support is naturally limited.

In the case of the flexibility and concision criteria, with the exception of IDEF, all the process modeling languages receive the same valuation. On the one hand, all of them might be adapted to different contexts of use. On the other hand, with a small set of modeling elements one might represent a large number of different organizational situations.

Generally speaking, with some minor differences, the ease of learning may be considered true to all of the process modeling languages. In fact, with the exception of IDEF, which can be a little bit more cumbersome, anyone can become a productive modeler after a few days of learning and experimentation with any of the languages.

Regarding the innovation inducer criterion, the process modeling language that we think is more capable to stimulate users in finding new solutions is RAD. Not being a formal language, RAD gives users the freedom to search for new ways of doing things and innovate. The other languages, more or less impose restrictions, and so reduce the creativity of modelers.

The evolutionary criterion tries to represent the probability of a language to be upgraded and improved in the future. There are no doubts that BPMN, EPC and 
UML-AD will evolve, integrating more and more features. In the case of IDEF, and particularly RAD, the perspectives of evolution are much more limited.

The collaborative work as a criterion to compare business process modeling languages will be more and more relevant in the future. Indeed, there are many situations during the execution of processes in which two or more people have to collaborate (synchronously or asynchronously) in order to execute some work. These situations have to be properly represented in process models. In this context, RAD has major advantages as it allows the representation of collaborative work very easily. BPMN, EPC and UML-AD also allow the representation of collaborative work, but not in a direct manner. Once again, IDEF completely misses the point because, as we mentioned earlier, it cannot represent the organizational perspective of processes.

\section{Comparative Framework Tool Development}

Having defined a framework to compare business process modeling languages, the next step is to develop a tool to implement this framework. To be useful, the tool has to support several requirements:

- First of all, the contents of the framework (Table 2) must be dynamic. Indeed, the current values of Table 2 are only the result of a limited study, in which the opinions of some authors, and our own opinions, are reflected. It would be convenient that the framework could evolve, incorporating the opinions of experienced business process modelers and analysts in order to improve its content. In that sense, at each moment the contents of Table 2 would represent the weighted opinions of specialists that have contributed to the framework until that moment;

- Second, the framework should be extensible in the sense that other business process modeling languages could be added to it, and also the list of criteria could be extended, if needed;

- Finally, the framework should assist users in choosing the most adequate process modeling language, taking into account the specificities of their BPM projects. To do that, in the course of choosing a language, users should have the possibility to give weights to each of the criteria, in order to best describe their needs in terms of process modeling.

A functional prototype, whose user interface, at the moment, is mainly in Portuguese, has already been developed, incorporating the three requirements above. It is available as a web portal which is open to the community of experts in process modeling languages, waiting to receive their contributions in order to improve the contents of the framework. So, the platform is prepared to continuously evolve and improve its content, giving solid results to users who need to choose a process modeling language, adequate to the characteristics of their BPM projects.

The next three figures present some of the user interactions with the web portal involved in the course of choosing a process modeling language. In the next figure (Fig.1) a user selected the criteria expressiveness (in Portuguese, Expressividade) and readability (in Portuguese, Legibilidade) as the more relevant criteria to his next BPM project. 


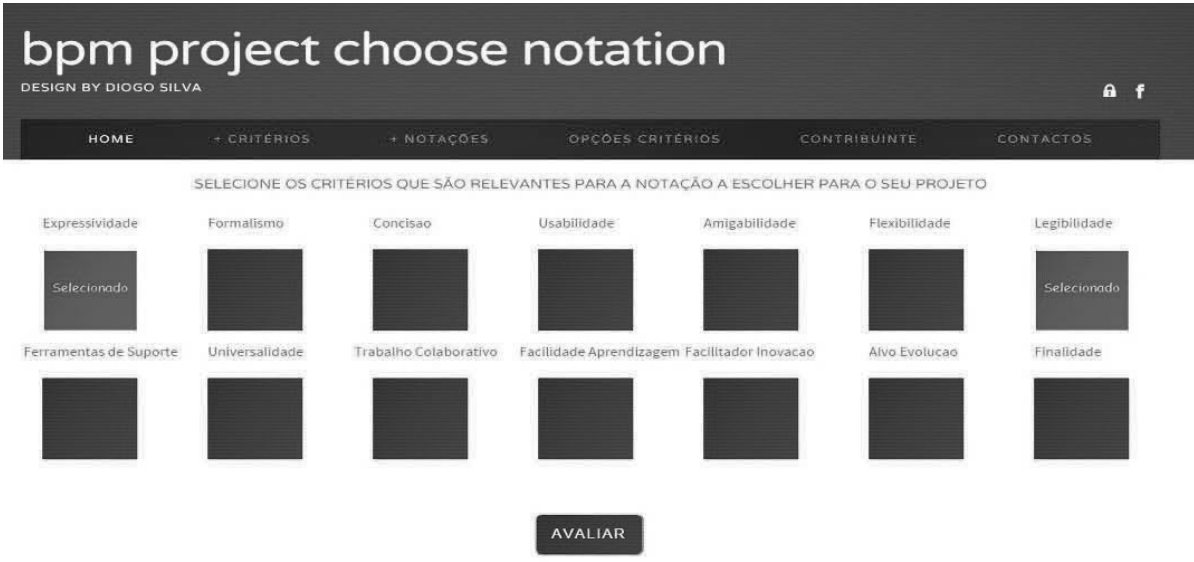

\section{๑) Uminho 2015. All rights reserved.}

Fig. 1. Selecting the relevant criteria

Next the user is invited to weight each one of the criteria selected in the previous step, choosing one of three values ( 1 - Low, 2 - Medium, 3 - High). This way a user indicates the level of importance attributed to each criterion. In Fig. 2, the expressiveness criterion waits to be characterized.

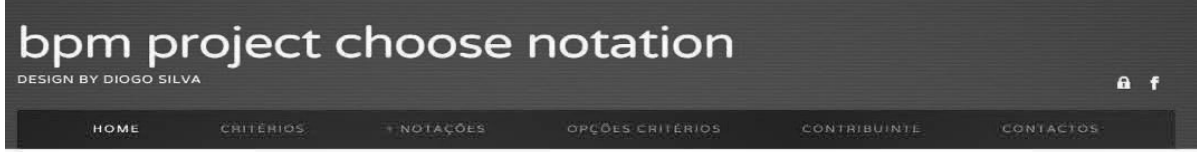

SELECIONE O NIVESS OUE PRETENDE.

EM TERMOS DE EXPRESSIVIDADE, OUAL O NIVEL OUEE NECESSARIO ATINGIR?

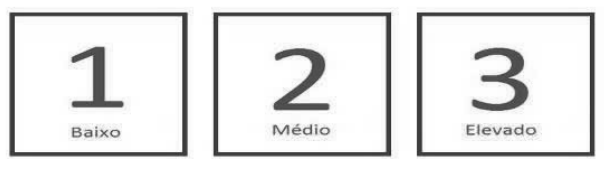

Fig. 2. Weighting the "expressiveness" criterion 
Finally, the results are presented (Fig. 3)

\section{bpm project choose notation}

DESIGN BY DIOGO SILVA

HOME

OPCOES CRITERIOS: CONTRIBUINTE

RESULTADOS
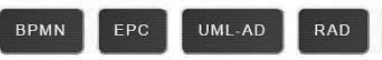

IDEFO

GRAFICO GERAL

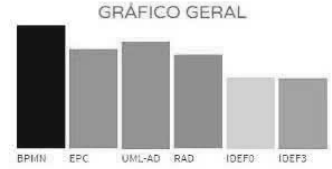

Fig. 3. Final results

In this example, having weighted the two criteria (expressiveness and readability) with high importance, BPMN results as the best process modeling language, followed successively by UML-AD, EPC, RAD and IDEF. One can also check the score of each modeling language by passing the cursor over the corresponding bar. The confidence level for each criterion (which depends on the number of existing contributions in the knowledge base at that time) is also displayed.

\section{Conclusions}

Due to the widespread recognition of the Business Process Management (BPM) approach, the modeling of business processes has recently gained increased importance. Nowadays, there is a considerable list of business process modeling languages one can use in the context of a BPM project. The fact is that each one of those languages has their own strengths and limitations. So, which one should we use in a specific BPM project?

In this paper, taking into account the available literature about business process modeling languages comparisons, complemented with our own experience in modeling business processes, we developed a framework that compares several languages (BPMN, EPC, IDEF, RAD and UML-AD) against a set of criteria. This 
framework evaluates quantitatively the level of support that each process modeling language offers to each criterion.

The goal of the comparative framework is to provide users with the means to select the most suitable business process modeling language, taking into account the characteristics of the BPM project. To facilitate the selection task we developed a tool that implements the comparative framework, allowing users to easily describe their needs in terms of the characteristics that the process modeling language should support.

The comparative framework is available as a web platform, open to users who need to select a process modeling language, and to modeling experts who might contribute with their evaluation to the content of the framework. In order to accommodate future requirements, the framework is completely extensible, allowing the addition of new process languages and criteria.

\section{References}

1. van der Aalst, W. M. P. (2013). Business Process Management: A Comprehensive Survey, ISRN Softw. Eng., vol., pp. 1-37.

2. Freund, J. \& Rücker, B. (2014). Real-Life BPMN: Using BPMN 2.0 to Analyze, Improve, and Automate Processes in Your Company (2nd Ed), CreateSpace Independent Publishing Platform.

3. Davis, R. (2008). ARIS Design Platform: Advanced Process Modelling and Administration, Springer.

4. Podeswa, H. (2009). UML for the IT Business Analyst (2nd Ed), Cengage Learning PTR.

5. Costin, B. \& Fox, C. (2004). Hybrid IDEF0 / IDEF3 Modelling of Business Processes : Syntax, Semantics and Expressiveness, Concurr. Eng., pp. 3-5.

6. Ould, M. (1995). Business Processes: Modelling and Analysis for Re-Engineering and Improvement, Wiley.

7. Heidari, F., Loucopoulos, P., Brazier, F. \& Barjis, J. (2012). A Unified View of Business Process Modelling Languages 1, Vol. 004.

8. Korherr, B. (2008). Business Process Modelling - Languages, Goals and Variabilities, Vienna University of Technology, PhD Thesis.

9. Mohammadi, M., \& Mukhtar, M. B. (2012). Business Process Modelling Languages in Designing Integrated Information System for Supply Chain Management. International Journal on Advanced Science Engineering Information Technology, 54-57.

10.Mili, H., Tremblay, G., Jaoude, G., Lefebvre, É., Elabed, L., \& El Boussaidi, G. (2010). Business process modeling languages: Sorting Through the Alphabet Soup, ACM Comput. Surv. vol. 43, no. 1, pp. 1-56.

11.Kelemen, Z. D., Kusters, R., Trienekens, J., \& Balla, K. (2013). Selecting a Process Modeling Language for Process Based Unification of Multiple Standards and Models, 1-14.

12.Aldin L., \& De Cesare, S. (2009). A comparative analysis of business process modelling techniques, UKAIS 2009, Oxford, UK, pp. 1-17.

13.Van Wel, R. (2013). Business Best practices in Agile software development, Leiden University, Master's Thesis. 